DOI: $10.17516 / 1997-1397-2020-13-5-583-595$

УДК 512.5

\title{
On the Equationally Artinian Groups
}

\author{
Mohammad Shahryari* \\ Department of Mathematics \\ College of Science \\ Sultan Qaboos University \\ Muscat, Oman \\ Javad Tayyebi ${ }^{\dagger}$ \\ Department of Pure Mathematics \\ Faculty of Mathematical Sciences \\ University of Tabriz \\ Tabriz, Iran
}

Received 26.05.2020, received in revised form 02.07.2020, accepted 16.08.2020

\begin{abstract}
In this article, we study the property of being equationally Artinian in groups. We define the radical topology corresponding to such groups and investigate the structure of irreducible closed sets of these topologies. We prove that a finite extension of an equationally Artinian group is again equationally Artinian. We also show that a quotient of an equationally Artinian group of the form $G[t]$ by a normal subgroup which is a finite union of radicals, is again equationally Artnian. A necessary and sufficient condition for an Abelian group to be equationally Artinian will be given as the last result. This will provide a large class of examples of equationally Artinian groups.
\end{abstract}

Keywords: algebraic geometry over groups, systems of group equations, radicals, Zariski topology, radical topology, equationally Noetherian groups, equationally Artinian groups.

Citation: M. Shahryari, J. Tayyebi, On the Equationally Artinian Groups, J. Sib. Fed. Univ. Math. Phys., 2020, 13(5), 583-595. DOI: 10.17516/1997-1397-2020-13-5-583-595.

In the mid-twentieth century, Alfred Tarski asked whether two arbitrary non-abelian free groups are elementary equivalent. To answer this question, it was necessary to investigate systems of equations over groups. Makanin and Razborov proved that the existence of solutions for systems of equations over free groups is a decidable problem and an algorithm to solve such systems of equation is discovered (Makanin-Razborov diagrams, see [10] and [14]). The work of Makanin and Razborov as well as many other mathematicians was the beginning of algebraic geometry over groups. Since then, this new area of algebra was the subject of important studies in group theory. The work of Baumslag, Myasnikov and Remeslennikov provides a complete account of this new subject, [1]. Positive solution to the problem of Tarski is discovered by Kharlampovich, Myasnikov and Sela at the the beginning of the recent century (see [7-9] and [15]). After that, many mathematicians investigated the algebraic geometry over general algebraic systems and this new area of algebra is now known as universal algebraic geometry. The reader can see the works of Daniyarova, Myasnikov, and Remeslennikov as well as the lecture notes of Plotkin as introduction to this branch, [3-6], and [13].

One of the very important notions in the algebraic geometry of groups (as well as other algebraic structures) is the property of being equationally Noetherian. Note that if $S$ is a system of equations over a group $A$, then we say that the system $S$ implies an equation $w \approx 1$, if every

\footnotetext{
*m.ghalehlar@squ.edu.om

$\dagger$ j.tayyebi@tabrizu.ac.ir

(C) Siberian Federal University. All rights reserved
} 
solution of $S$ in $A$ is also a solution of $w \approx 1$. This gives us an equational logic over the group $A$ which is not in general similar to the first order logic. For example, the compactness theorem may fails in this equational logic. There are examples of groups such that the compactness for the systems of equations fails (see [1] and [5] for some examples). In some groups, every system of equations is equivalent to a finite subsystem, such groups are called equationally Noetherian. Free groups, Abelian groups, linear groups over Noetherian rings and torsion-free hyperbolic groups are equationally Noetherian. To see interesting properties of this types of groups, the reader can consult [11] and [16]. This kind of groups have very important roles in algebraic geometry of groups. There are many equivalent conditions for the property of being equationally Noetherian, for example, it is known that a group $A$ has this property, if and only if, for any natural number $n$, every descending chain of algebraic sets in $A^{n}$ is finite. According to this equivalent condition, in [11] and [12], the dual property of being equationally Artinian is defined. A group $A$ is equationally Artinian, if and only if, for any natural number $n$, every ascending chain of algebraic sets in $A^{n}$ is finite. In [12], many equivalent conditions to this property is given.

In 1997, Baumslag, Myasnikov, and Romankov proved two important theorems about equationally Noetherian groups: first, they showed that a virtually equationally Noetherian group is equationally Noetherian. They also showed that quotient of an equationally Noetherian group by a normal subgroup which is a finite union of algebraic sets, is again equationally Noetherian (see [2]). In this Article we prove similar results for the case of equationally Artinian groups. These results will provide a large class of examples for equationally Artinian groups. Also, we study irreducible closed subsets of the radical topology in the case of equationally Artinian groups and we obtain a necessary and sufficient condition for an Abelian group to be equationally Artinian.

\section{Preliminaries}

Let $G$ be an arbitrary group and suppose that $X=\left\{x_{1}, \ldots, x_{n}\right\}$ is a finite set of variables. Consider the free product $G[X]=G * F[X]$, where $F[X]$ is the free group over $X$. Every element $w \in G[X]$ corresponds to an equation $w \approx 1$, which is called a group equation with coefficients from $G$. If $w=w\left(x_{1}, \ldots, x_{n}, g_{1}, \ldots, g_{m}\right) \in G[X]$, then the expression $w \approx 1$ is a $G$-equation with coefficients $g_{1}, \ldots, g_{m} \in G$. Suppose $H$ is a group which contains $G$ as a distinguished subgroup. Then we say that $H$ is a $G$-group. A tuple $\bar{h}=\left(h_{1}, \ldots, h_{n}\right) \in H^{n}$ is called a root of the equation $w \approx 1$, if

$$
w\left(h_{1}, \ldots, h_{n}, g_{1}, \ldots, g_{m}\right)=1 .
$$

An arbitrary set of $G$-equations is called a system of equation with coefficients from $G$. The set of all common roots of the elements of $S$ in $H$ is called the corresponding algebraic set of $S$ and denoted by $V_{H}(S)$. Clearly, the intersection of a non-empty family of algebraic sets is again an algebraic set but the same is not true for unions of algebraic sets. If we define a closed subset of $H^{n}$ to be an arbitrary intersection of finite unions of algebraic sets, then we get a topology on $H^{n}$, which is known as Zariski topology.

For a subset $E \subseteq H^{n}$, we define the corresponding radical $\operatorname{Rad}(E)$ to be the set of all elements $w \in G[X]$ such that every element of $E$ is a solution of $w \approx 1$. This is a normal subgroup of $G[X]$ which is called the radical of $E$ and the quotient group $\Gamma(E)=G[X] / \operatorname{Rad}(E)$ is called the coordinate group of $E$. Similarly, for a system $S$, we define its radical to be $\operatorname{Rad}_{H}(S)=\operatorname{Rad}\left(V_{H}(S)\right)$. This is the largest system of $G$-equations equivalent to $S$ over $H$. The corresponding coordinate group is $\Gamma_{H}(S)=G[X] / \operatorname{Rad}_{H}(S)$. It is proved that the study of coordinate groups is equivalent to the study of Zariski topology, i.e. algebraic geometry of $H$ reduces to the study coordinate groups, [1]. 
A $G$-group $H$ is called $G$-equationally Noetherian, if for every system $S$, there exists a finite subsystem $S_{0}$, such that $V_{H}(S)=V_{H}\left(S_{0}\right)$. Such $G$-groups have important role in the study of algebraic geometry over $G$-groups. There are two extremal cases: if $G=1$, we say that $H$ is 1-equationally Noetherian or equationally Noetherian without coefficients, and if $G=H$, then we say that $H$ is equationally Noetherian (or equationally Noetherian in Diophantine sense). It is proved that a 1-equationally Noetherian finitely generated group is equationally Noetherian as well, [1]. The class of equationally Noetherian groups is very large, containing all Free groups, Abelian groups, linear groups over Noetherian rings and torsion-free hyperbolic groups are equationally Noetherian. It is not hard to see that the following statements are equivalent for a $G$-group $H$ :

i- $H$ is $G$-equationally Noetherian.

ii- the Zariski topology on $H^{n}$ is Noetherian for all $n$.

iii- every chain of coordinate groups and proper epimorphisms

$$
\Gamma\left(E_{1}\right) \rightarrow \Gamma\left(E_{2}\right) \rightarrow \Gamma\left(E_{3}\right) \rightarrow \cdots
$$

is finite.

The authors of [2] proved two important theorems about equationally Noetherian groups. The first theorem shows that a finite extension of an equationally Noetherian group is again equationally Noetherian. The second theorem says that if $G$ is equationally Noetherian and $N$ is a normal subgroup which is a finite union of algebraic sets (in Diophantine case), then $G / N$ is also equationally Noetherian. In this article, we are dealing with the dual notion, the property of being equationally Artinian and we prove the similar statements for this type of groups.

\section{Equationally Artinian groups}

Equationally Artinian algebras are introduced in [11] and [12]. In this section, we review this notion for the case of $G$-groups. We say that a $G$-group $H$ is $G$-equationally Artinian, if for any $n$, every ascending chain of algebraic sets in $H^{n}$ terminates. This is not equivalent to the property of being Artinian for the Zariski topology, instead we define a new topological space which becomes Noetherian if $H$ is equationally Artinian. Suppose

$$
T=\left\{u \operatorname{Rad}(E): E \subseteq H^{n}, u \in G[X]\right\} .
$$

Note that for arbitrary cosets $u \operatorname{Rad}(E)$ and $v \operatorname{Rad}(F)$, if their intersection is non-empty, then for an arbitrary element $w \in u \operatorname{Rad}(E) \cap v \operatorname{Rad}(F)$, we have $w \operatorname{Rad}(E)=u \operatorname{Rad}(E)$ and $w \operatorname{Rad}(F)=v \operatorname{Rad}(F)$. Hence $u \operatorname{Rad}(E) \cap v \operatorname{Rad}(F)=w(\operatorname{Rad}(E) \cap \operatorname{Rad}(F))=w \operatorname{Rad}(E \cup F)$. This shows that the intersection of two cosets of radicals, is again a coset of a radical subgroup (or it is empty). The set $T$ is a subbasis of closed sets of a topology on the set $G[X]$ which is called the radical topology on $G[X]$ corresponding to $H$ (this topology is finer than the previous one defined in [12], in fact the subbasis introduced in [12] is a fundamental system of closed sets containing the identity of $G[X])$. Every closed set in $G[X]$ is an arbitrary intersection of finite unions of cosets of the form $u \operatorname{Rad}(E)$, with $E \subseteq H^{n}$ and $u \in G[X]$. In [12], it is proved that the following statements are equivalent for a $G$-group $H$ :

i- $H$ is $G$-equationally Artinian.

ii- for any $n$ and any subset $E \subseteq H^{n}$, there exists a finite subset $E_{0} \subseteq E$, such that $\operatorname{Rad}(E)=\operatorname{Rad}\left(E_{0}\right)$.

iii- the corresponding radical topology over $G[X]$ is Noetherian. 
Remark 1. The proof is essentially the same as in [12], but since we used here our enhanced definition of finer radical topology, so we show that why the proof remains unchanged. We only need to show that for a $G$-group $H$, being $G$-equationally Artinian is equivalent to the property of being Noetherian for the corresponding radical topology on $G[X]$. So, let $H$ be $G$-equationally Artinian and

$$
T=\left\{u \operatorname{Rad}(E): E \subseteq H^{n}, u \in G[X]\right\} .
$$

We first prove that $T$ satisfies the descending chain condition. Suppose

$$
u_{1} \operatorname{Rad}\left(E_{1}\right) \supseteq u_{2} \operatorname{Rad}\left(E_{2}\right) \supseteq u_{3} \operatorname{Rad}\left(E_{3}\right) \supseteq \cdots
$$

is a descending chain of elements of $T$. Then we have also the following chain

$$
\operatorname{Rad}\left(E_{1}\right) \supseteq \operatorname{Rad}\left(E_{2}\right) \supseteq \operatorname{Rad}\left(E_{3}\right) \supseteq \cdots .
$$

Therefore,

$$
V_{H}\left(\operatorname{Rad}\left(E_{1}\right)\right) \subseteq V_{H}\left(\operatorname{Rad}\left(E_{2}\right)\right) \subseteq V_{H}\left(\operatorname{Rad}\left(E_{3}\right)\right) \subseteq \cdots,
$$

and this later chain terminates, as $H$ is $G$-equationally Artinian. So, for some $k$, we have

$$
V_{H}\left(\operatorname{Rad}\left(E_{k}\right)\right)=V_{H}\left(\operatorname{Rad}\left(E_{k+1}\right)\right)=V_{H}\left(\operatorname{Rad}\left(E_{k+2}\right)\right)=\cdots .
$$

Taking one more radical, we get

$$
\operatorname{Rad}\left(E_{k}\right)=\operatorname{Rad}\left(E_{k+1}\right)=\operatorname{Rad}\left(E_{k+2}\right)=\cdots .
$$

This shows that

$$
u_{k} \operatorname{Rad}\left(E_{k}\right)=u_{k+1} \operatorname{Rad}\left(E_{k+1}\right)=u_{k+2} \operatorname{Rad}\left(E_{k+2}\right)=\cdots,
$$

and hence $T$ satisfies the descending chain condition. Now, let $T_{1}$ be the set of all finite unions of elements of $T$ and $T_{2}$ be the set of all arbitrary intersection of elements of $T_{1}$. Note that $T_{2}$ is the set of all closed subsets of $G[X]$ with respect to the radical topology. We show that $T_{1}$ also satisfies the descending chain condition. Suppose that

$$
M_{1}=u_{1} \operatorname{Rad}\left(E_{1}\right) \cup \ldots \cup u_{m} \operatorname{Rad}\left(E_{m}\right), M_{2}=v_{1} \operatorname{Rad}\left(F_{1}\right) \cup \ldots \cup v_{k} \operatorname{Rad}\left(F_{k}\right)
$$

are sets in $T_{1}$ and $M_{2} \subset M_{1}$. For every $i \leqslant m$ and $j \leqslant k$, we have $u_{i} \operatorname{Rad}\left(E_{i}\right) \cap v_{j} \operatorname{Rad}\left(F_{j}\right) \subseteq$ $u_{i} \operatorname{Rad}\left(E_{i}\right)$. Hence we can gain a tree with root vertex $u_{i} \operatorname{Rad}\left(E_{i}\right)$ and with a unique edge from the root to every proper subset $u_{i} \operatorname{Rad}\left(E_{i}\right) \cap v_{j} \operatorname{Rad}\left(F_{j}\right) \subset u_{i} \operatorname{Rad}\left(E_{i}\right)$. Suppose there exists a strictly descending chain of subsets in $T_{1}$ :

$$
M_{1} \supset M_{2} \supset M_{3} \supset \cdots .
$$

As we mentioned, we obtain a tree for any inclusion $M_{i} \supset M_{i+1}$, such that each vertex is a finite intersection of sets in $T$, hence each vertex is in $T$ itself, since as we saw above, the non-empty intersections of a finite number of elements from $T$ are again belong to $T$. Since each vertex is connected to only finite number of other vertices, so each vertex has finite degree. So, every path corresponds to a strictly descending chain of radicals and since $H$ is $G$-equationally Artinian, so the path is finite. By the well-known König's lemma of graph theory, this implies that the graph is finite. Therefore the above chain is also finite. So $T_{1}$ satisfies the descending chain condition and is closed under finite intersection.

Now, we prove that $T_{2}$ satisfies the descending chain condition too. Suppose $\bigcap_{i=1}^{\infty} R_{i}$ is an infinite intersection of elements of $T_{1}$. Then we have the following chain:

$$
R_{1} \supseteq R_{1} \cap R_{2} \supseteq R_{1} \cap R_{2} \cap R_{3} \supseteq \cdots .
$$


Since $T_{1}$ satisfies descending chain condition and is closed under finite intersection, so the chain terminates. Therefore

$$
\exists k: R_{1} \cap R_{2} \cap \ldots \cap R_{k}=\bigcap_{i=1}^{\infty} R_{i} .
$$

Hence, every infinite intersection of subsets of $T_{1}$ is in fact a finite intersection in $T_{1}$ and so it belongs to $T_{1}$. Consequently, we have $T_{2}=T_{1}$ and hence it satisfies the descending chain condition. This shows that the radical topology on $G[X]$ is Noetherian. The proof of the converse statement is trivial.

By $(E A)_{G}$, we denote the class of all $G$-equationally Artinian $G$-groups, by $(E A)_{1}$, the class of 1-equationally Artinian groups and $E A$ will be used for the class of Equationally Artinian groups (Diophantine case where $G=H$ ). In this article, we first prove the following theorem.

Theorem 1. Let $G \in E A$ be torsion-free and $E \subseteq G^{n}$ be an algebraic set. Then the set $\operatorname{Rad}(E)$ is irreducible and all irreducible closed subset of $G[X]$ is a coset of some radical.

Our main tool to prove this result is a well-known theorem of B. Neumann which says that if a group covered by a finite set of cosets of subgroups, then at least one of those subgroups has finite index. This result of Neumann also will be used to prove the following result.

Theorem 2. Let $G \in E A$ be torsion-free and $E \subseteq G^{n}$ be a non-empty algebraic set with $\operatorname{Rad}(E) \neq G[X]$. Then the interior of $\operatorname{Rad}(E)$ is empty.

Note that every Noetherian topological space has finite number of irreducible components. In the case of a torsion-free equationally Artinian group $G$, the space $G[X]$ has a unique irreducible component, say $G[X]$ itself. Theorem 2, also shows that if $G \in E A$ is torsion-free, then $G[X]$ is connected. We will prove the converse for coefficient-free case.

Theorem 3. Let $G \in(E A)_{1}$. Then $G$ is torsion-free if and only if, $F[X]$ is connected.

Note that there are many equationally Noetherian groups which are not equationally Artinian, for example, the additive group $\mathbb{Q} / \mathbb{Z}$, the multiplicative group of complex numbers, the quasicyclic groups $\mathbb{Z}_{p^{\infty}}$ (see also Theorem 8). Many other groups like non-Abelian free groups and torsion-free hyperbolic groups are failed to be equationally Artinian (as they are domains and every equationally Artinian domain is finite). It must be said that, at the time of writing this paper, we don't know if there is equationally Artinian group which is not equationally Noetherian. But, both classes are included in a larger class of groups which we call equationally semi-Noetherian. A group $G$ has this property, if for every system of equations $S \subseteq G[X]$, almost every finite subset $T \subseteq S$ can be omitted solving the system over $G$, i.e. there exists a finite subset $S_{0} \subseteq S$ such that for all other finite subset $T \subseteq S \backslash S_{0}$, we have $V_{G}(S)=V_{G}(S \backslash T)$. Clearly, every equationally Noetherian group has this property. We will prove,

Theorem 4. If $G \in E A$, then $G$ is equationally semi-Noetherian.

Our next theorem concerns about an important relation between the classes $(E A)_{1}$ and $(E A)_{G}$. We prove,

Theorem 5. Let $G$ be a finitely generated group and let $H$ be a $G$-group. If $H \in(E A)_{1}$, then $H \in(E A)_{G}$, and as a result, any finitely generated element of $(E A)_{1}$ is equationally Artinian.

In our sixth theorem, we deal with finite extensions of equationally Artinian groups. We prove,

Theorem 6. Let a group A contains a finite index subgroup $H$ which is equationally Artinian. Then $A$ is also equationally Artinian. 
This theorem enables us to conclude that any virtually finitely generated Abelian group is equationally Artinian as well as any finite extension of the additive group of any field. This gives us a large class of examples of such groups. This theorem is $E A$-version of the similar theorem in $[2]$.

Note that the quotient of an equationally Artinian group is not necessarily equationally Artinian (for example the group $\mathbb{Q} / \mathbb{Z}$ ), but, there exists an important situation, the quotient in which, has this property. Our nest result concerns with these situations. Note that in this theorem, we use the group $G[t]=G *\langle t\rangle$.

Theorem 7. Let $G$ be an arbitrary group such that $G[t]$ is equationally Artinian. Let $R$ be a normal subgroup of $G[t]$ which is closed in the radical topology of $G[t]$. Then $G[t] / R$ is also equationally Artinian.

Finally, we will show that an Abelian group $G$ is equationally Artinian, if and only if, it has finite number of periods: let $p(G)$ be the set of orders of torsion elements of $G$. We will prove,

Theorem 8. An Abelian group $G$ is equationally Artinian, if and only if, $p(G)$ is finite.

\section{The proofs}

Proof. (Theorem 1 and 2) Suppose $G$ is equationally Artinian. Let $Y$ be an irreducible closed subset of $G[X]$. Since $Y$ is a finite union of cosets of the form $u \operatorname{Rad}(E)$, so $W=u \operatorname{Rad}(E), \operatorname{for}$ some algebraic set $E \subseteq G^{n}$ and $u \in G[X]$. Now, for an algebraic set $E$, we show that $\operatorname{Rad}(E)$ is irreducible. Note that every closed subset of $\operatorname{Rad}(E)$ has the form $v_{1} \operatorname{Rad}\left(L_{1}\right) \cup \ldots \cup v_{p} \operatorname{Rad}\left(L_{p}\right)$, where $v_{i} \in G[X]$ and $E \subseteq L_{i}$. Now, if $\operatorname{Rad}(E)$ can be written as a union of two closed subsets, then we have

$$
\operatorname{Rad}(E)=\bigcup_{i=1}^{m} u_{i} \operatorname{Rad}\left(K_{i}\right),
$$

for some elements $u_{i} \in G[X]$ and algebraic sets $K_{i}$ with $E \subseteq K_{i}$. It is a well-known theorem of B. Neumann which says that if a group is covered by a finite number of cosets of subgroups, then at least one of those subgroups has finite index. So, we have for example $[\operatorname{Rad}(E)$ : $\left.\operatorname{Rad}\left(K_{1}\right)\right]<\infty$. Suppose now that $G$ is torsion-free and $\operatorname{Rad}\left(K_{1}\right) \neq \operatorname{Rad}(E)$. Choose an element $w \in \operatorname{Rad}(E)$, such that for some $\bar{a} \in K_{1}$, we have $w(\bar{a}) \neq 1$. Then, for all non-zero integers $k$ we have also $w^{k}(\bar{a}) \neq 1$ and hence all cosets $w^{j} \operatorname{Rad}\left(K_{1}\right),(1 \leqslant j)$, are distinct. This shows that $\operatorname{Rad}(E)=\operatorname{Rad}\left(K_{1}\right)$ and so $\operatorname{Rad}(E)$ is irreducible. Note that in any Noetherian space, there is a finite number of maximal irreducible sets (irreducible components) and in the case of $G[X]$, $\operatorname{Rad}(\varnothing)=G[X]$ is the only irreducible component.

Now, we show that the interior of $\operatorname{Rad}(E)$ is empty for any $E \neq \varnothing$. Let an open set $G[X] \backslash \bigcup_{j=1}^{m} w_{j} \operatorname{Rad}\left(E_{j}\right)$ be contained in $\operatorname{Rad}(E)$. Then we have

$$
G[X]=\operatorname{Rad}(E) \cup \bigcup_{j=1}^{m} w_{j} \operatorname{Rad}\left(E_{j}\right),
$$

and again using the theorem of Neumann, some of these subgroups has finite index, which is shows that $G[X] \backslash \bigcup_{j=1}^{m} w_{j} \operatorname{Rad}\left(E_{j}\right)=\varnothing$. Hence $\operatorname{Rad}(E)$ has empty interior.

Proof. (Theorem 3) In this proof, we denote the coefficient-free radical of a subset $E \in G^{n}$ by $\operatorname{Rad}^{0}(E)$, i.e.

$$
\operatorname{Rad}^{0}(E)=\{w \in F[X]: \forall \bar{a} \in E w(\bar{a})=1\}
$$


Suppose first that $F[X]$ is not connected. Then we have

$$
F[X]=\bigcup_{i=1}^{m} u_{i} \operatorname{Rad}^{0}\left(E_{i}\right)
$$

for some elements $u_{i} \in F[X]$ and subsets $E_{i} \subseteq G^{n}$, where $m \geqslant 2$ is minimal. Again by the theorem of Neumann, there is an index $i$ such that $\left[F[X]: \operatorname{Rad}^{0}\left(E_{i}\right)\right]$ is finite and is not equal to 1 by the minimality of $m$. This shows that the coefficient-free coordinate group $\Gamma\left(E_{i}\right)$ is finite and non-trivial. But, we know that this coordinate group embeds inside a direct power of $G$. So, $G$ is not torsion-free, a contradiction.

Conversely, assume that $G$ is not torsion-free. Let $a \in G$ be a non-trivial element of finite order $m$ and put $\bar{a}=(a, 1, \ldots, 1) \in G^{n}$. Let $w=x_{1}$. Then clearly, $w^{m} \in \operatorname{Rad}^{0}(\bar{a})$. Consider the subgroup $\langle w\rangle \operatorname{Rad}^{0}(\bar{a})$. This subgroup contains all elements $x_{1}, \ldots, x_{n}$, and so we have $F[X]=\langle w\rangle \operatorname{Rad}^{0}(\bar{a})$. Now, we have

$$
F[X]=\bigcup_{i=0}^{m-1} w^{i} \operatorname{Rad}^{0}(\bar{a}),
$$

and hence, $F[X]$ is not connected.

We now prove Theorem 4 . Note that the proof can be applied for arbitrary algebraic structures as well.

Proof. (Theorem 4) Suppose $G$ is equationally Artinian and $S \subseteq G[X]$ is an infinite system. For simplicity, assume $S=\left\{v_{1}, v_{2}, v_{3}, \ldots\right\}$. We have the ascending chain

$$
V_{G}(S)=V_{G}\left(v_{1}, v_{2}, v_{3}, \ldots\right) \subseteq V_{G}\left(v_{2}, v_{3}, v_{4}, \ldots\right) \subseteq V_{G}\left(v_{3}, v_{4}, v_{5}, \ldots\right) \subseteq \cdots .
$$

This chain terminates as $G$ is equationally Artinian, so there exists $k$ such that

$$
V_{G}\left(v_{k}, v_{k+1}, v_{k+2}, \ldots\right)=V_{G}\left(v_{k+1}, v_{k+2}, v_{k+3}, \ldots\right)=V_{G}\left(v_{k+2}, v_{k+3}, v_{k+4}, \ldots\right) \cdots .
$$

This shows that

$$
\bigcap_{j \geqslant k} V_{G}\left(v_{j}\right)=\bigcap_{j \geqslant k+1} V_{G}\left(v_{j}\right)=\bigcap_{j \geqslant k+2} V_{G}\left(v_{j}\right)=\cdots,
$$

and hence

$$
\begin{aligned}
V_{G}(S) & =V_{G}\left(v_{1}, \ldots, v_{k-1}\right) \cap \bigcap_{j \geqslant k} V_{G}\left(v_{j}\right) \\
& =V_{G}\left(v_{1}, \ldots, v_{k-1}\right) \cap \bigcap_{j \geqslant k+1} V_{G}\left(v_{j}\right) \\
& =V_{G}\left(v_{1}, \ldots, v_{k-1}\right) \cap \bigcap_{j \geqslant k+2} V_{G}\left(v_{j}\right) .
\end{aligned}
$$

In other words, this argument shows that the algebraic sets $V_{G}\left(v_{j}\right)$ can be drop in the intersection for $j \geqslant k$. Let $S_{0}=\left\{v_{1}, \ldots, v_{k-1}\right\}$. Then by this argument, for any finite subset $T \subseteq S \backslash S_{0}$, we have $V_{G}(S)=V_{G}(S \backslash T)$.

Proof. (Theorem 5) Let $a_{1}, \ldots, a_{k}$ be a finite set of generators for the group $G$. Suppose $E \subseteq H^{n}$. We prove that there exists a finite subset $E_{0} \subseteq E$, such that $\operatorname{Rad}_{G}(E)=\operatorname{Rad}_{G}\left(E_{0}\right)$ (note that, here $\operatorname{Rad}_{G}$ denotes the radical with coefficient in $G$ ). Let $S=\operatorname{Rad}_{G}(E) \subseteq G[X]$. Every element of $S$ has the form

$$
w=w\left(x_{1}, \ldots, x_{n}, a_{1}, \ldots, a_{k}\right) .
$$


We replace every coefficient $a_{i}$ by a new variable $y_{i}$, and then a coefficient-free system of equations $S(\bar{x}, \bar{y})$ appears. Let $T=E \times\left\{\left(a_{1}, \ldots, a_{k}\right)\right\} \subseteq H^{n+k}$. Now, since $H \in(E A)_{1}$, so there is a finite subset $T_{0} \subseteq T$, such that $\operatorname{Rad}_{1}(T)=\operatorname{Rad}_{1}\left(T_{0}\right)$. Clearly, we have $T_{0}=E_{0} \times\left\{\left(a_{1}, \ldots, a_{k}\right)\right\}$, for some finite subset $E_{0} \subseteq E$. Obviously, $S(\bar{x}, \bar{y}) \subseteq \operatorname{Rad}_{1}(T)$. Let $u(\bar{x}, \bar{y}) \in \operatorname{Rad}_{1}(T)$. Then for all $\bar{e} \in E$, we have $u(\bar{e}, \bar{a})=1$, so $u(\bar{x}, \bar{a}) \in \operatorname{Rad}_{G}(E)$, and therefore $u \in S(\bar{x}, \bar{y})$. This proves that $S(\bar{x}, \bar{y})=\operatorname{Rad}_{1}(T)$, and hence $S(\bar{x}, \bar{y})=\operatorname{Rad}_{1}\left(T_{0}\right)$.

Now, we show that $S(\bar{x}, \bar{a})=\operatorname{Rad}_{G}\left(E_{0}\right)$. Suppose $w(\bar{x}, \bar{a}) \in S(\bar{x}, \bar{a})$. For any $\bar{e} \in E_{0}$, we have $w(\bar{e}, \bar{a})=1$, so $w(\bar{x}, \bar{a}) \in \operatorname{Rad}_{G}\left(E_{0}\right)$. Conversely, if $w(\bar{x}, \bar{a}) \in \operatorname{Rad}_{G}\left(E_{0}\right)$, then for $w(\bar{x}, \bar{y}) \in \operatorname{Rad}_{1}\left(T_{0}\right)=\operatorname{Rad}_{1}(T)$, and this shows that $w(\bar{x}, \bar{a}) \in \operatorname{Rad}_{G}(E)=S(\bar{x}, \bar{a})$. This proves that $\operatorname{Rad}_{G}(E)=\operatorname{Rad}_{G}\left(E_{0}\right)$ and hence $H \in(E A)_{G}$.

Theorem 5, enables us to prove that every finitely generated Abelian group belongs to the class $E A$ (we also can deduce this from Theorem 8). Here we give an elementary proof which shows the infinite cyclic group is equationally Artinian.

Lemma 1. Let $H=\langle a\rangle$ be infinite cyclic group. Then $H$ is equationally Artinian.

Proof. We first show that $H \in(E A)_{1}$. Let $E \subseteq H^{n}$. Every element of $E$ has the form $\bar{e}=$ $=\left(a^{j_{1}}, \ldots, a^{j_{n}}\right)$ for some integers $j_{1}, \ldots, j_{n}$. Let $w=x_{1}^{\alpha_{1}} x_{2}^{\alpha_{2}} \ldots x_{n}^{\alpha_{n}} \in \operatorname{Rad}_{1}(E)$. Then $w(\bar{e})=1$ and hence $a^{j_{1} \alpha_{1}+\cdots+j_{n} \alpha_{n}}=1$. This shows that

$$
\operatorname{Rad}_{1}(E)=\bigcap_{j_{1}, \ldots, j_{n}}\left\{x_{1}^{\alpha_{1}} x_{2}^{\alpha_{2}} \ldots x_{n}^{\alpha_{n}}:\left(a^{j_{1}}, \ldots, a^{j_{n}}\right) \in E, j_{1} \alpha_{1}+\cdots+j_{n} \alpha_{n}=0\right\} .
$$

Suppose

$$
E=\left\{\left(a^{j_{1}^{(1)}}, \ldots, a^{j_{n}^{(1)}}\right),\left(a^{j_{1}^{(2)}}, \ldots, a^{j_{n}^{(2)}}\right),\left(a^{j_{1}^{(3)}}, \ldots, a^{j_{n}^{(3)}}\right), \ldots\right\} .
$$

Suppose $S$ is the following set of equations

$$
j_{1}^{(t)} \alpha_{1}+\cdots+j_{n}^{(t)} \alpha_{n}=0, \quad(t=1,2,3, \ldots) .
$$

Since the additive group $\mathbb{Z}$ is equationally Noetherian, so there exists a finite subset $S_{0} \subseteq S$, such that $V_{\mathbb{Z}}(S)=V_{\mathbb{Z}}\left(S_{0}\right)$. Suppose $S_{0}$ consists of the equations

$$
j_{1}^{(t)} \alpha_{1}+\cdots+j_{n}^{(t)} \alpha_{n}=0 \quad(t=1,2, \ldots, m) .
$$

Let $E_{0}=\left\{\left(a^{j_{1}^{(1)}}, \ldots, a^{j_{n}^{(1)}}\right),\left(a^{j_{1}^{(2)}}, \ldots, a^{j_{n}^{(2)}}\right), \ldots,\left(a^{j_{1}^{(m)}}, \ldots, a^{j_{n}^{(m)}}\right)\right\}$. Then we have obviously, $\operatorname{Rad}_{1}(E)=\operatorname{Rad}_{1}\left(E_{0}\right)$. This shows that $H$ is 1-equationally Artinian and hence by Theorem 2, it belongs to $E A$.

Now, we show that any direct product of finitely many element of $(E A)_{1}$ is again in $(E A)_{1}$. This will prove that every finitely generated Abelian group belongs to $(E A)_{1}$ and hence to $E A$.

Lemma 2. Suppose $A$ and $B$ are equationally Artinian (1-equationally Artinian). Then so is $A \times B$.

Proof. For a number $n$ and a subset $E \subseteq(A \times B)^{n}$, suppose that

$$
E=\left\{c_{i}=\left(u_{1}^{i}, u_{2}^{i}, \ldots, u_{n}^{i}\right): i \in I\right\},
$$

where $I$ is an index set. We have $u_{i}^{j}=\left(a_{i}^{j}, b_{i}^{j}\right)$, for some $a_{i}^{j} \in A$ and $b_{i}^{j} \in B$. Now, let

$$
T=\left\{t_{i}=\left(a_{1}^{i}, a_{2}^{i}, \ldots, a_{n}^{i}\right): i \in I\right\},
$$

and

$$
S=\left\{s_{i}=\left(b_{1}^{i}, b_{2}^{i}, \ldots, b_{n}^{i}\right): i \in I\right\} .
$$


Since $A$ and $B$ are equationally Artinian, so there are two finite subsets $T_{0} \subseteq T$ and $S_{0} \subseteq S$, such that

$$
\operatorname{Rad}_{A}(T)=\operatorname{Rad}_{A}\left(T_{0}\right), \operatorname{Rad}_{B}(S)=\operatorname{Rad}_{B}\left(S_{0}\right) .
$$

Suppose for example $T_{0}=\left\{t_{1}, \ldots, t_{l}\right\}$ and $S_{0}=\left\{s_{1}, \ldots, s_{k}\right\}$ and $k \geqslant l$. Suppose $t_{i}=\left(a_{1}^{i}, \ldots, a_{n}^{i}\right)$ and $s_{i}=\left(b_{1}^{i}, \ldots, b_{n}^{i}\right)$. Using these elements, we can define a finite subset

$$
E_{0}=\left\{c_{i}=\left(\left(a_{1}^{i}, b_{1}^{i}\right), \ldots,\left(a_{n}^{i}, b_{n}^{i}\right): 1 \leqslant i \leqslant l\right\},\right.
$$

such that $\operatorname{Rad}(E)=\operatorname{Rad}\left(E_{0}\right)$. This shows that $A \times B$ is equationally Artinian.

Summarizing, we have

Corollary 1. Every finitely generated Abelian group is equationally Artinian.

There are also infinitely generated Abelian groups which are equationally Artinian: let $K$ be a field and consider its additive group $H=(K,+)$. Every equation with coefficient in $K$ has the form $a_{1} x_{1}+\cdots+a_{n} x_{n}=b$ for some elements $a_{1}, \ldots, a_{n} \in \mathbb{Z}, b \in K$, so the corresponding algebraic set is an affine subspace of $K^{n}$. This shows that every ascending chain of algebraic sets terminates and hence $H$ is equationally Artinian. However, some Abelian groups are not equationally Artinian. For example, consider the additive group $H=\mathbb{Q} / \mathbb{Z}$. Let

$$
E=\left\{\frac{1}{p}+\mathbb{Z}: p=\text { prime }\right\} \subseteq H^{1} .
$$

If $w(x)=m x+\left(\frac{a}{b}+\mathbb{Z}\right) \in \operatorname{Rad}(E)$, then for any prime $p$, we have $w\left(\frac{1}{p}+\mathbb{Z}\right)=\mathbb{Z}$, and this means that for any prime $p, \frac{m}{p}+\frac{a}{b} \in \mathbb{Z}$, which is not true. Another example is the quasi-cyclic groups $G=\mathbb{Z}_{p^{\infty}}$, for prime numbers $p$. This is because, the ascending chain of algebraic sets $V_{G}\left(x^{p^{n}} \approx 1\right),(n \geqslant 1)$ does not terminate (this fact will be used in the proof of Theorem 8 ).

Before proving Theorem 6, we introduce some notations from [2]. Let a group $A$ be the semidirect product of a finite subgroup $T$ and a normal subgroup $H$. Assume that $T=\left\{t_{1}=1, t_{2}, \ldots, t_{k}\right\}$. Let $w\left(x_{1}, \ldots, x_{n}, g_{1}, \ldots, g_{m}\right)$ be a group word with coefficients in $A$ and $v \in A^{n}$. We can express $v$ uniquely in the form $v=\left(s_{1} h_{1}, \ldots, s_{n} h_{n}\right)$ with $s_{i} \in T$ and $h_{i} \in H$. We also have $g_{i}=r_{i} b_{i}$ for unique elements $r_{i} \in T$ and $b_{i} \in H$. Define the map $\lambda: A^{n} \rightarrow T^{n}$ by $\lambda(v)=\left(s_{1}, \ldots, s_{n}\right)$ and

$$
\bar{w}\left(x_{1}, \ldots, x_{n}\right)=w\left(x_{1}, \ldots, x_{n}, r_{1}, \ldots, r_{m}\right) .
$$

Note that $\bar{w}$ is an element of $T[X]$ which depends only on $w$. For any $1 \leqslant i \leqslant n$ and $1 \leqslant j \leqslant k$, define $h_{i j}=t_{j}^{-1} h_{i} t_{j} \in H$. Denote the tuple

$$
\left(h_{11}, \ldots, h_{1 k}, \ldots, h_{n 1}, \ldots, h_{n k}\right)
$$

by $v^{\prime}$. Consider the new variables $y_{i j}$ for $1 \leqslant i \leqslant n$ and $1 \leqslant j \leqslant k$. In [2], it is proved that there exists a unique element

$$
w_{v}^{\prime} \in H\left[y_{11} \ldots, y_{1 k}, \ldots, y_{n 1}, \ldots, y_{n k}\right],
$$

such that $w(v)=\bar{w}(\lambda(v)) w_{v}^{\prime}\left(v^{\prime}\right)$, and $w_{v}^{\prime}$ depends only on the value of $\lambda(v)$. As a result, it is shown that $v \in A^{n}$ is a root of $w \approx 1$, if and only if, $\lambda(v)$ is a root of $\bar{w} \approx 1$ and $v^{\prime}$ is a root of $w_{v}^{\prime} \approx 1$. We are now ready to prove Theorem 6 . 
Proof. (Theorem 6) Replacing $H$ by its core, we can suppose that $H$ is a normal subgroup of $A$ with finite index. Let $T=A / H$. Then $A$ embeds into the wreath product $H \imath T$. Recall that this wreath product is the semidirect product of $T$ and $H^{|T|}$. We know that (Lemma 2), $H^{|T|}$ is equationally Artinian and any subgroup of an equationally Artinian group is again equationally Artinian. So, it is enough to prove our theorem using the further assumption $A=T H$, with $T$ finite, $H$ normal and $T \cap H=1$. We will use all the above notations.

Suppose $E \subseteq A^{n}$ is an algebraic set and $S=\operatorname{Rad}_{A}(E)$. We must show that there exists a finite subset $E_{0} \subseteq E$, such that $\operatorname{Rad}_{A}\left(E_{0}\right)=S$. Let $\bar{S}=\{\bar{w}: w \in S\}$ (see the above discussion). Suppose

$$
V_{T}(\bar{S})=\left\{v_{1}, \ldots, v_{d}\right\} \subseteq T^{n} .
$$

For any $1 \leqslant i \leqslant d$, put $L_{i}=V_{H}\left(S_{v_{i}}^{\prime}\right) \subseteq H^{n k}$. Here $S_{v_{i}}^{\prime}$ denotes the set of all $w_{v_{i}}^{\prime}$, such that $w \in S$. Define also

$$
K_{i}=\left\{\bar{h} \in H^{n}:(\bar{h})^{\prime} \in L_{i}\right\} \subseteq H^{n} .
$$

We have $\left(K_{i}\right)^{\prime} \subseteq H^{n k}$ and since $H$ is equationally Artinian, there exists a finite subset $K_{i}^{0} \subseteq K_{i}$, such that

$$
\operatorname{Rad}_{H}\left(\left(K_{i}^{0}\right)^{\prime}\right)=\operatorname{Rad}_{H}\left(\left(K_{i}\right)^{\prime}\right) .
$$

Assume that $E_{0}=\cup_{i=1}^{d} v_{i} K_{i}^{0} \subseteq A^{n}$. We show that $E_{0} \subseteq E$. Let $v_{i} \bar{h} \in E_{0}$. Then $\bar{h} \in K_{i}$ and hence

$$
\bar{S}\left(\lambda\left(v_{i} \bar{h}\right)\right)=\bar{S}\left(v_{i}\right)=1,
$$

and

$$
S_{v_{i}}^{\prime}\left(\left(v_{i} \bar{h}\right)^{\prime}\right) \in S_{v_{i}}^{\prime}\left(L_{i}\right)=1 .
$$

This means that $v_{i} \bar{h} \in V_{A}(S)=E$. Therefore $E_{0} \subseteq E$.

Now, we claim that $\operatorname{Rad}_{A}\left(v_{i} K_{i}^{0}\right)=\operatorname{Rad}_{A}\left(v_{i} K_{i}\right)$. To prove this claim, assume that $w$ belongs to the left hand side. Then $w\left(v_{i} K_{i}^{0}\right)=1$ and hence $w^{\prime}\left(\left(v_{i} K_{i}^{0}\right)^{\prime}\right)=1$. This shows that $w_{v_{i}}^{\prime} \in \operatorname{Rad}_{H}\left(\left(v_{i} K_{i}^{0}\right)^{\prime}\right)$. Recall that, by the definition of the map $v \mapsto v^{\prime}$, we have $\left.\left(v_{i} K_{i}^{0}\right)^{\prime}\right)=\left(K_{i}^{0}\right)^{\prime}$ and hence $w_{v_{i}}^{\prime} \in \operatorname{Rad}_{H}\left(\left(K_{i}^{0}\right)^{\prime}\right)=\operatorname{Rad}_{H}\left(\left(K_{i}\right)^{\prime}\right)=\operatorname{Rad}_{H}\left(\left(v_{i} K_{i}\right)^{\prime}\right)$. Therefore, for any $\bar{h} \in K_{i}$, we have $w_{v_{i}}^{\prime}\left(\left(v_{i} \bar{h}\right)^{\prime}\right)=1$, and since in the same time $\bar{w}\left(\lambda\left(v_{i} \bar{h}\right)\right)=1$, we have $w\left(v_{i} K_{i}\right)=1$. This proves the claim.

We now, prove that $\operatorname{Rad}_{A}\left(E_{0}\right)=\operatorname{Rad}_{A}(E)$. Let $w$ be an element of the left hand side and $v \in E$. We have $S(v)=1$ and

$$
w \in \bigcap_{i=1}^{d} \operatorname{Rad}_{A}\left(v_{i} K_{i}^{0}\right) .
$$

Note that $v=\lambda(v) \bar{h}$, for some $\bar{h} \in H^{n}$. We have $\bar{S}(\lambda(v))=1$, so there is an index $i$ such that $\lambda(v)=v_{i}$. Therefore, $v=v_{i} \bar{h}$. On the other side, since $S_{v}^{\prime}\left(V^{\prime}\right)=1$, so

$$
1=S_{v}^{\prime}\left(v^{\prime}\right)=S_{v_{i}}^{\prime}\left(\left(v_{i} \bar{h}\right)^{\prime}\right) \text {. }
$$

Hence, $\left(v_{i} \bar{h}\right)^{\prime} \in L_{i}$, and therefore $\bar{h} \in K_{i}$. Now, by the above claim, we have

$$
w \in \operatorname{Rad}_{A}\left(v_{i} K_{i}^{0}\right)=\operatorname{Rad}_{A}\left(v_{i} K_{i}\right),
$$

and hence $w(v)=1$. This shows that $w \in \operatorname{Rad}_{A}(E)$.

Theorem 6 shows that any virtually finitely generated Abelian group is equationally Artinian as well as any finite extension of the additive group of any field. This gives us a large class of examples of such groups. We now come to Theorem 7. Note that the similar theorem ([2]) for the equationally Noetherian case deals with the Zarizki topology of $G^{1}$ and its closed normal subgroups. The dual case here deals with the radical topology of $G[t]$ and its closed normal subgroups. 
Proof. Assume that

$$
R=\bigcup_{i=1}^{m} \operatorname{Rad}_{G}\left(K_{i}\right)
$$

where $K_{i} \subseteq G$. Note that $G$ is equationally Artinian as $G[t]$ is so. Hence every $K_{i}$ can be chosen finite. Let $H=G[t] / R$ be not equationally Artinian. Hence there exists a number $n$ and a subset $E \in H^{n}$ such that $\operatorname{Rad}_{H}(E) \neq \operatorname{Rad}_{H}\left(E_{0}\right)$, for any finite subset $E_{0} \subseteq E$. Assume that $e_{0} \in E$ is an arbitrary element. As $\operatorname{Rad}_{H}(E) \neq \operatorname{Rad}_{H}\left(\left\{e_{0}\right\}\right)$, there exist elements $f_{1} \in \operatorname{Rad}_{H}\left(\left\{e_{0}\right\}\right)$ and $e_{1} \in E$, such that $f_{1}\left(e_{1}\right) \neq 1$. Similarly, we have $\operatorname{Rad}_{H}(E) \neq \operatorname{Rad}_{H}\left(\left\{e_{0}, e_{1}\right\}\right)$, so there exist elements $f_{2} \in \operatorname{Rad}_{H}\left(\left\{e_{0}, e_{1}\right\}\right)$ and $e_{2} \in E$, such that $f_{2}\left(e_{2}\right) \neq 1$. Repeating this argument, we obtain two infinite sequences

$$
\begin{gathered}
f_{1}, f_{2}, f_{3}, \ldots \in H[X], \\
e_{0}, e_{1}, e_{2}, \ldots \in E,
\end{gathered}
$$

such that for any $i, f_{i}\left(e_{0}\right)=f_{i}\left(e_{1}\right)=\cdots=f_{i}\left(e_{i-1}\right)=1$, but $f_{i}\left(e_{i}\right) \neq 1$. Note that, here $X=\left\{x_{1}, \ldots, x_{n}\right\}$ and so every element of $H[X]$ is a word in $t$ and elements of $X$ with coefficients in $G$. Suppose $q: G[t, X] \rightarrow H[X]$ is the canonical map sending elements of $G$ to their cosets, and fixing elements of $X$ and the element $t$. Suppose also that $\psi:(G[t])^{n} \rightarrow H^{n}$ is the map

$$
\psi\left(u_{1}, \ldots, u_{n}\right)=\left(u_{1} R, \ldots, u_{n} R\right) .
$$

Choose a pre-image $\bar{f}_{i}$ for $f_{i}$ under $q$ and a pre-image $\bar{e}_{i}$ for $e_{i}$ under $\psi$. Hence, we have $\bar{f}_{i} \in G[t, X]$ and $\bar{e}_{i} \in(G[t])^{n}$. For any $i$, we have $f_{i}\left(e_{0}\right)=1$, so $\bar{f}_{i}\left(\bar{e}_{0}\right) \in R$. This shows that, there exists an infinite sequence of numbers

$$
i_{1}(0)<i_{2}(0)<i_{3}(0)<\cdots,
$$

and a number $1 \leqslant p_{0} \leqslant m$, such that

$$
\bar{f}_{i_{1}(0)}\left(\bar{e}_{0}\right), \bar{f}_{i_{2}(0)}\left(\bar{e}_{0}\right), \bar{f}_{i_{3}(0)}\left(\bar{e}_{0}\right), \ldots \in \operatorname{Rad}_{G}\left(K_{p_{0}}\right) .
$$

Equivalently, this shows that for all $s$, we have

$$
\bar{f}_{i_{s}(0)} \in \operatorname{Rad}_{G[t]}\left(\bar{e}_{0}\left(K_{p_{0}}\right)\right) .
$$

By a similar argument, we obtain an infinite subsequence of $\left\{i_{s}(0)\right\}$ of the form

$$
i_{1}(1)<i_{2}(1)<i_{3}(1)<\cdots,
$$

and a number $1 \leqslant p_{1} \leqslant m$, such that for all $s$, we have

$$
\bar{f}_{i_{s}(1)} \in \operatorname{Rad}_{G[t]}\left(\bar{e}_{1}\left(K_{p_{1}}\right)\right) .
$$

We continue this process to find an infinite subsequence

$$
i_{1}(k)<i_{2}(k)<i_{3}(k)<\cdots,
$$

of the previous sequence, and a number $1 \leqslant p_{k} \leqslant m$, such that

$$
\bar{f}_{i_{s}(k)} \in \operatorname{Rad}_{G[t]}\left(\bar{e}_{k}\left(K_{p_{k}}\right)\right),
$$

for all $s$. Note that all sets $\bar{e}_{i}\left(K_{p_{i}}\right)$ are finite as $K_{i}$ 's are finite. Let

$$
K=\bigcup_{i=0}^{\infty} \bar{e}_{i}\left(K_{p_{i}}\right) \subseteq(G[t])^{n}
$$


By assumption, $G[t]$ is equationally Artinian, so there exists an index $l$, such that

$$
\operatorname{Rad}_{G[t]}(K)=\operatorname{Rad}_{G[t]}\left(\bigcup_{i=0}^{l} \bar{e}_{i}\left(K_{p_{i}}\right)\right) .
$$

Assume that $j>l$. Then for any $s$, we have

$$
\bar{f}_{i_{s}(j)} \in \bigcap_{i=1}^{l} \operatorname{Rad}_{G[t]} \bar{e}_{i}\left(K_{p_{i}}\right)=\operatorname{Rad}_{G[t]}(K) .
$$

Suppose $k=i_{1}(j)$. Then $\bar{f}_{k} \in \operatorname{Rad}_{G[t]}\left(\bar{e}_{k}\left(K_{p_{k}}\right)\right)$, and hence $\bar{f}_{k}\left(\bar{e}_{k}\right) \in \operatorname{Rad}_{G}\left(K_{p_{k}}\right) \subseteq R$. This shows that $f_{k}\left(e_{k}\right)=1$, a contradiction. Hence $H$ is equationally Artinian.

Finally, we give a proof for Theorem 8.

Proof. (Theorem 8) We first, show that a divisible Abelian group $G$ is equationally Artinian, if and only if, it is torsion-free. Recall that a divisible Abelian group has the form $G=\mathbb{Q}^{I} \oplus$ $\sum_{p \in J} \mathbb{Z}_{p^{\infty}}$, for an index set $I$ and a set $J$ of prime numbers. If $G$ is torsion-free then $G=\mathbb{Q}^{I}$, and since the additive group of rationales is equationally Artinian, so is $G$. Now, suppose that $G$ is equationally Artinian but is not torsion-free. Then for some prime $p$, we have $\mathbb{Z}_{p} \infty \leqslant$, and this implies that $\mathbb{Z}_{p^{\infty}}$ is equationally Artinian, a contradiction.

Now, suppose that $G$ is an arbitrary Abelian group. Assume that $p(G)$ is finite. We know that $G=\operatorname{Tor}(G) \oplus G_{1}$, where $\operatorname{Tor}(G)$ is the torsion part of $G$ and $G_{1}$ is a torsion-free subgroup. We know that $G_{1}$ can be embedded in some divisible Abelian group and hence it is equationally Artinian. The torsion part has finite exponent and hence can be written in the form $\operatorname{Tor}(G)=\bigoplus_{m \in p(G)} \mathbb{Z}_{m}^{I_{m}}$, where for all $m \in p(G)$, an index set $I_{m}$ is associated. Clearly, every component $\mathbb{Z}_{m}^{I_{m}}$ is equationally Artinian and since $p(G)$ is finite, so the direct sum is also so. This shows that $G \in E A$.

Finally, suppose that in a group $G$, the set $p(G)$ is infinite. Let $m_{1}<m_{2}<m_{3}<\cdots$ be elements of $p(G)$ such that for all $i$ the integer $m_{1} m_{2} \ldots m_{i-1}$ is not divisible by $m_{i}$. For any $i$, assume that $a_{i}$ is an element of order $m_{i}$. Consider the ascending chain

$$
V_{G}\left(x^{m_{1}} \approx 1\right) \subseteq V_{G}\left(x^{m_{1} m_{2}} \approx 1\right) \subseteq V_{G}\left(x^{m_{1} m_{2} m_{3}} \approx 1\right) \subseteq \cdots
$$

This chain does not terminate, because for any $i$, we have

$$
a_{1}, \ldots, a_{i} \in V_{G}\left(x^{m_{1} \ldots m_{i}} \approx 1\right)
$$

but $a_{i+1}$ does not belong to it. Therefore $G$ is not equationally Artinian.

\section{References}

[1] G.Baumslag, A.Myasnikov, V.Remeslennikov, Algebraic geometry over groups, I. Algebraic sets and ideal theory, J. Algebra, 2191999, 16-79.

[2] G.Baumslag, A.Myasnikov, V.Romankov, Two theorems about equationally Noetherian groups, J. Algebra, 1997, 194, 654-64.

[3] E.Daniyarova, A.Myasnikov, V.Remeslennikov, Algebra and Discrete Mathamatics, 1(2008), 80-112. DOI: 10.1142/9789812793416_0007

[4] E.Daniyarova, A.Myasnikov, V.Remeslennikov, Algebraic geometry over algebraic structures, II: Fundations, J. Math. Sci., 185(2012), no. 3, 389-416. 
[5] E.Daniyarova, A.Myasnikov, V.Remeslennikov, Algebraic geometry over algebraic structures, III: Equationally noetherian property and compactness, South. Asian Bull. Math., 35(2011), no. 1, 35-68.

[6] E.Daniyarova, A.Myasnikov, V.Remeslennikov, Algebraic geometry over algebraic structures, IV: Equatinal domains and co-domains, Algebra and Logic, 49(2011), no. 6, 483-508.

[7] O.Kharlampovich, A.Myasnikov, Tarski's problem about the elementary theory of free groups has a psitive solution, E.R.A. of AMS, 4(1998), 101-108.

[8] O.Kharlampovich, A.Myasnikov, Irreducible affine varieties over a free group. I: irreducibility of quadratic equations and Nullstellensatz, J. Algebra, 200(1998), no. 2, 472-516.

[9] O.Kharlampovich, A.Myasnikov, The elemntary theory of free non-abelian groups, J. Algebra, 302(2006), 451-552.

[10] G.Makanin, Equations in free groups, Math. USSR-Izv., 21(1982), no. 3, 483-546.

[11] P.Modabberi, M.Shahryari, Compactness conditions in universal algebraic geometry, Algebra and Logic, 55(2016), no. 2, 146-172.

[12] P.Modabberi, M.Shahryari, On the equational Artinian algebras, Siberian Electronic Mathematical Reports, 13(2016), 875-881.

[13] B.Plotkin, Seven lectures in universal algebraic geometry, preprints, Arxiv.

[14] A.Razborov, On systems of equations in free groups, Math. USSR-Izv., 1982, 25, no. 1, $115-162$.

[15] Z.Sela, Diophantine geometry over groups: I-X, preprints, Arxiv.

[16] M.Shahryari, A.Shevliyakov, Direct products, varieties, and compacness conditions, Groups Complex, Cryptol, 9(2017), no. 2, 159-166.

\section{Об эквивалентно артиновых группах}

Мохаммед Шахриари

Математический факультет, Колледж Науки

Университет Султана Кабуса

Мускат, Оман

Джавад Тайеби

Кафедра чистой математики, Факультет математических наук Университет Тебриза

Тебриз, Иран

Аннотация. В этой статье мы изучаем свойство быть артиновым в группах. Определяем радикальную топологию, соответствующую таким группам, и исследуем структуру неприводимых замкнутых множеств этих топологий. Докажем, что конечное расширение уравновешенно артиновой группы снова уравновешенно артиново. Мы также показываем, что частное от артиновоуравновешенной группы вида $G[t]$ по нормальной подгруппе, являющейся конечным объединением радикалов, опять-таки уравновешенно артново. В качестве последнего результата будет дано необходимое и достаточное условие, чтобы абелева группа была эквивалентно артиновой. Это обеспечит большой класс примеров уравновешенно артиновых групп.

Ключевые слова: алгебраическая геометрия над группами, системы групповых уравнений, радикалы, топология Зариского, радикальная топология, нетеровы группы, эквационально артиновы группы. 